\title{
GROWTH PROCESSES OF ZnTe EPILAYERS DEPOSITED BY MBE ON GaAs(100) VICINAL SURFACES - STUDIES BY STATIC AND DYNAMIC RHEED
}

\author{
J. Sadowski, Z. DziUba \\ Institute of Physics, Polish Academy of Sciences \\ Al. Lotników 32/46, 02-668 Warszawa, Poland \\ AND M.A. Herman \\ Institute of Vacuum Technology, Długa 44/50, 00-241 Warszawa, Poland
}

\begin{abstract}
Static and dynamic reflection high energy electron diffraction (RHEED) has been applied for studying the initial growth processes of $\mathrm{ZnTe}$ crystallized by molecular beam epitaxy (MBE) on vicinal surfaces of GaAs(100) substrates. Atomically smooth $\mathrm{ZnTe}$ epilayers have been grown by MBE when in situ thermal desorption of the substrate protecting oxide layer was performed in the ultra high vacuum environment of the vacuum growth chambcr just before the growth of $\mathrm{ZnTe}$ started. By gradual increasing of the substrate temperature of the crystallized $\mathrm{ZnTe}$ epilayers from $300^{\circ} \mathrm{C}$ to $420^{\circ} \mathrm{C}$, when recording the RHEED intensity oscillations at these and eleven intermittent temperatures, it has been shown that the transition from the $2 \mathrm{D}$-nucleation growth mechanism to the step-flow growth mechanism of $\mathrm{ZnTe}$ occurs at $410^{\circ} \mathrm{C}$. Measuring periods of RHEED intensity oscillations recorded during the MBE growth processes it has been demonstrated that the growth rate of $\mathrm{ZnTe}$ at constant fluxes of the constituent elements decreases with increasing temperature from $0.37 \mathrm{ML} / \mathrm{s}$ at $300^{\circ} \mathrm{C}$ to $0.22 \mathrm{ML} / \mathrm{s}$ at $400^{\circ} \mathrm{C}$.
\end{abstract}

PACS numbers: $81.15 . \mathrm{Gh}, 61.14 . \mathrm{Hg}$

\section{Introduction}

It is well known [1] that atomically smooth epilayers deposited by MBE on off-oriented $\mathrm{GaAs}(100)$ substrates (exhibiting steps and plane parallel terraces, called vicinal surfaces) grow by two different mechanisms. The first one is defined by 2D-nucleation process occurring on the vicinal surfaces, while the second is defined by surface migration of adsorbed constituent atoms of the epilayer towards the steps. When the migrating atoms reach the step, they become incorporated 
(at the step) into the crystal lattice of the epilayer. This is the step-flow growth mechanism. The occurrence of the definite growth mechanism depends on the temperature of the substrate and the structural parameters of the vicinal surfaces. We have used static and dynamic reflection high energy electron diffraction (RHEED) for studies of the initial growth processes [2] of $\mathrm{ZnTe}$ on vicinal surfaces of $\operatorname{GaAs}(100)$ substrates. The following two problems have been investigated in details: (i) control over transition from 2D-nucleation growth mechanism to step-flow growth mechanism, (ii) growth rates of $\mathrm{ZnTe}$ epilayers in dependence on temperature.

\section{Substrate preparation procedure}

The pre-growth preparation procedure of the substrate surface plays a crucial role in controlling the initial growth process of epilayers in heteroepitaxy. In our experiments with $\mathrm{ZnTe}$ growth by MBE three different procedures of pre-growth preparation of the $2^{\circ}$ off-oriented $\operatorname{GaAs}(100)$ substrate surface have been investigated. As a qualification criterion for the preparation procedure, the static RHEED diffractograms have been used. It is well known [2] that atomically smooth surfaces are characterized by a streaky RHEED pattern, preferably with pronounced surface reconstruction features. On the other side, when the RHEED pattern characteristic for the investigated surface is spotty, the surface is rough on atomic scale.

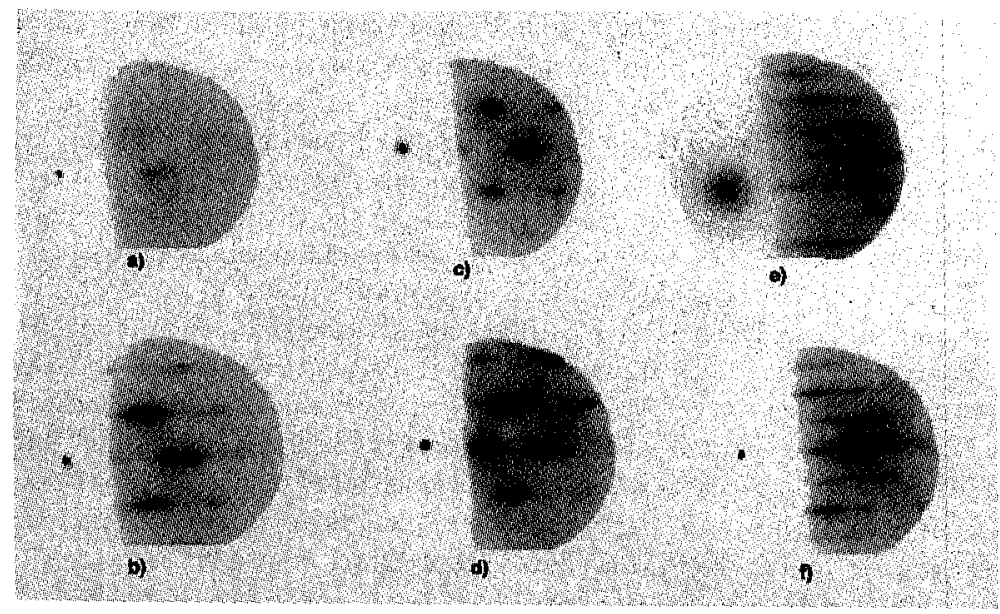

Fig. 1. RHEED diffractograms taken from (a)-(d) $2^{\circ}$ off-oriented $\mathrm{GaAs}(100)$ substrates, pre-growth prepared according to different procedures; (e) $2000 \mathrm{~nm}$ thick $\mathrm{ZnTe}$ epilayer grown on the substrate (d); (f) $2000 \mathrm{~nm}$ thick $\mathrm{ZnTe}$ epilayer grown on the substrate (b).

Figure 1 shows RHEED diffractograms taken from $\mathrm{GaAs}(100)$ off-oriented substrate surfaces prepared according to the following procedures: (a) epiready surface (Sumitomo), covered with a protection oxide layer (at room temperature), (b) epiready surface (Sumitomo) after in situ thermally desorbed oxide at $580^{\circ} \mathrm{C}$, 
(c) substrate produced by the Institute of Electronic Materials Technology, Warsaw (ITME), etched according to the following procedure: etching in $\mathrm{NH}_{4} \mathrm{OH}+\mathrm{H}_{2} \mathrm{O}_{2}$ $+\mathrm{H}_{2} \mathrm{O}, 20: 7: 973$ for $3 \mathrm{~min}$, rinsing in deionized water for $3 \mathrm{~min}$, etching in $\mathrm{NH}_{4} \mathrm{OH}$ $+\mathrm{H}_{2} \mathrm{O}, 1: 10$ for $15 \mathrm{~s}$ drying in pure nitrogen; RHEED after pre-growth annealing in ultra high vacuum (UHV) for $15 \mathrm{~min}$ at temperature increasing until $580^{\circ} \mathrm{C}$, and (d) etched ITME substrate after annealing for $30 \mathrm{~min}$ at $580^{\circ} \mathrm{C}$ in $\mathrm{Te}$ flux $\left(\mathrm{Te}_{2}\right.$ beam equivalent pressure was equal to $10^{-7} \mathrm{hPa}$ ). Two $\mathrm{ZnTe}$ epilayers $2000 \mathrm{~nm}$ thick, have been grown on the two substrate surfaces, structurally best according to diffractograms shown in Fig. 1 (in situ deoxidized Sumitomo (Fig. 1b) and etched ITME with subsequent annealing in Te (Fig. 1d)). In both cases the MBE growth has been performed at $300^{\circ} \mathrm{C}$ when delivering the constituent elements from two effusion cells charged with solid $\mathrm{Te}$ and $\mathrm{Zn}$, respectively. RHEED diffractograms taken from the epilayers are shown in Fig. 1e and Fig. 1f. Analyzing the RHEED diffractograms one may conclude that the pre-growth preparation procedure that allows one to obtain the ZnTe epilayers of the best quality on GaAs $2^{\circ}$ off-oriented substrates is this one, in which the in situ thermal desorption of the protecting oxide has been employed. Here (Fig. 1f), a clear $(2 \times 2)$ reconstruction of the $\mathrm{Zn}$-stabilised $\mathrm{ZnTe}$ surface may be recognised.

\section{Growth mechanism dependence on the growth temperature}

It is possible to explore the growth dynamics of MBE by monitoring temporal variations in the intensity of various features in the RHEED patterns (dynamic RHEED). Neave et al. [3] examined RHEED oscillations occurring during the growth of $\mathrm{GaAs}(100)$ substrates at increase of the substrate temperatures. The principal finding of this work was the progressing reduction in the intensity of the RHEED oscillations until a critical temperature when there are no oscillations. This effect has been interpreted as a transition from the low-temperature growth by 2D-nucleation on the vicinal surfaces to a high-temperature mode where the increased mobility of the adsorbed constituents of the grown compound ( $\mathrm{Ga}$ and As) leads to direct incorporation of migrating adatoms into the growth front at the terrace edges. Thus, in a high temperature regime, the growth proceeds by step propagation (step-flow mode), for which the mean surface step density remains approximately constant, in contrast to the 2D-growth mode in which the step density varies as on a flat surface of an exactly oriented $\mathrm{GaAs}(100)$ substrate. The recorded sequence of measured intensity oscillations of the half-order feature of the RHEED pattern, occurring during MBE growth of ZnTe on Sumitomo epiready deoxidized GaAs substrates at increasing temperatures is shown in Fig. 2. One may clearly see from the presented oscillograms that the relevant transition temperature for $\mathrm{ZnTe}$ was equal to $410^{\circ} \mathrm{C}$. To our best knowledge this is the first ever published experimental result concerning the MBE growth mode transition for $\mathrm{ZnTe}$ grown on vicinal surfaces of $\mathrm{GaAs}(100)$ substrates.

Having recorded RHEED intensity oscillations during the MBE growth, one may estimate the growth rate of the epilayer. It has been originally shown by Neave et al. [4] that the period of RHEED intensity oscillations exactly corresponds to the growth rate of a single molecular $(\mathrm{Ga}+\mathrm{As})$ monolayer of $\mathrm{MBE}$ grown $\mathrm{GaAs}$. 

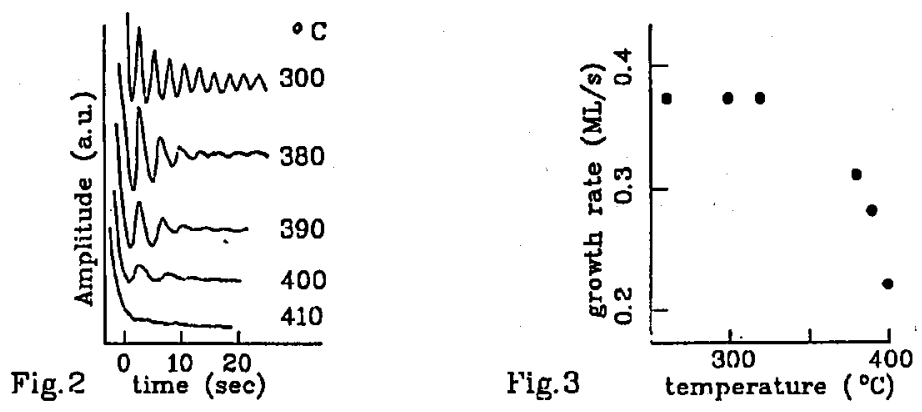

Fig. 2. Oscillations of the $1 / 2$ order RHEED feature recorded during MBE growth of ZnTe epilayers.

Fig. 3. Dependence of the growth rate of $\mathrm{MBE}$ grown $\mathrm{ZnTe}$ on the substrate temperature.

This principle holds also for other material systems [2]. We have found from data presented in Fig. 2 that the growth rate of $\mathrm{ZnTe}$ crystallized on vicinal surfaces of $\mathrm{GaAs}(100)$ substrates shown in Fig. 3 decreases with increasing temperature from $0.37 \mathrm{ML} / \mathrm{s}$ at $300^{\circ} \mathrm{C}$ to $0.22 \mathrm{ML} / \mathrm{s}$ at $400^{\circ} \mathrm{C}$.

\section{Conclusions}

Growing by MBE ZnTe on substrate surfaces prepared by in situ thermal deoxidation we have obtained atomically smooth heterolayers with $(2 \times 2)$ surface reconstruction (Zn-stabilized surface). ZnTe heteroepilayers grow on vicinal GaAs substrates by the 2D-nucleation mechanism at the substrate temperatures below $410^{\circ} \mathrm{C}$, while over this temperature the growth occurs according to the step-flow mechanism. Analysing the temperature dependence of periods of the RHEED intensity oscillations occurring during $\mathrm{ZnTe}$ growth, the growth rate decrease with increase in the substrate temperature, caused by desorption processes more intense at the higher substrate temperature, has been evidenced.

\section{Acknowledgments}

This work has been supported by the State Committee for Scientific Research (Republic of Poland) through grants No. 8-8037-92-03, 3-3713-92-03 and PBZZ011/P4/93/01.

\section{References}

[1] S. Clarke, D.D. Vvedensky, Phys. Rev. Lett. 58, 2235 (1987).

[2] M.A. Herman, H. Sitter, Molecular Beam Epitaxy-Fundamentals and Current Status, Springer, Berlin 1989.

[3] J.H. Neave, P.J. Dobson, B.A. Joyce, J. Zhang, Appl. Phys. Lett. 47, 100 (1985).

[4] J.II. Neave, B.A. Joyce, P.J. Dobson, N. Norton, Appl. Phys. A 31, 1 (1983). 